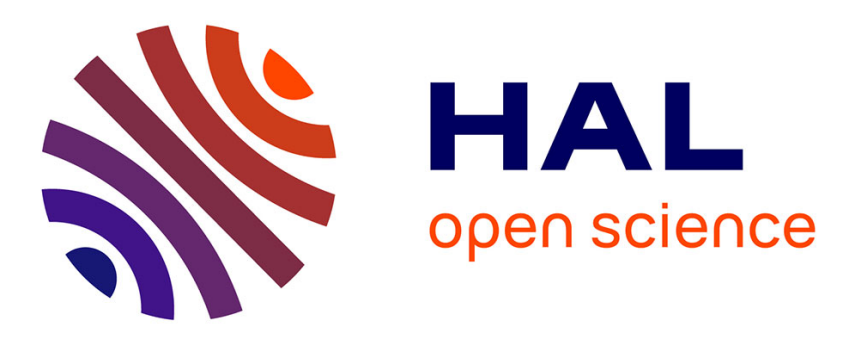

\title{
Autonomy based modeling for the simulation of ocean remote sensing
}

Marc Parenthoën, Zakaria Belemaalem

\section{To cite this version:}

Marc Parenthoën, Zakaria Belemaalem. Autonomy based modeling for the simulation of ocean remote sensing. OCEAN - IEEE, 2008, Quebec, Canada. 10.1109/OCEANS.2008.5152089 . hal-01260546

\section{HAL Id: hal-01260546 \\ https://hal.science/hal-01260546}

Submitted on 26 Jan 2016

HAL is a multi-disciplinary open access archive for the deposit and dissemination of scientific research documents, whether they are published or not. The documents may come from teaching and research institutions in France or abroad, or from public or private research centers.
L'archive ouverte pluridisciplinaire HAL, est destinée au dépôt et à la diffusion de documents scientifiques de niveau recherche, publiés ou non, émanant des établissements d'enseignement et de recherche français ou étrangers, des laboratoires publics ou privés. 


\title{
Autonomy based modeling for the simulation of ocean remote sensing
}

\author{
Marc Parenthoën and Zakaria Belemaalem \\ Laboratory of Computer Science for Complex Systems (LISyC - EA 3883) \\ Brest National Engineer School (ENIB), European Centre for Virtual Reality (CERV) \\ 25, rue Claude Chappé, F-29280 Plouzané, France \\ Email: \{parenthoen,belemaalem\}@enib.fr
}

\begin{abstract}
This article presents the simulation structure for the MODENA project [1] and illustrates what should become an original dynamical model for the reflectivity map of the sea surface. From our point of view, MODENA challenges to find accurate models for simulating dynamically interaction between sea state, sonar, radar and ship, while keeping physical coherency during the whole observation scenario (several tens of minutes), including human participation in this virtual reality system. To deal with such a complexity, as it is impossible and inappropriate to compute dynamically hydrodynamic, electromagnetic and acoustic equations on an accurate grid of points or mesh, MODENA simulation structure aims to use an autonomy based approach: the model of each phenomenon (wave, breaking, group, ship, wind, stream, transmitter, receiver...) involved in the simulation is seen as an autonomous entity, including an autonomy of time, space and scale. These models are combined and animated using enaction-based multi-agents simulation. In such simulation, computer activity is optimised at places where and when interaction occurs within revelant scale, according to choosen models. Furthermore, to add new phenomena or to modify existing models do not make previous work obsolete, as one has only to characterise interactions of new phenomena with previously defined models. This modeling approach facilitates interaction between research teams involved in the MODENA project, as the whole simulation results from the set of autonomized models developed by each team in parallel. Such a virtual laboratory should help for example to better distinguish the signal of breaking from the signal of small boats, in the dynamical signature of sea-states.
\end{abstract}

\section{INTRODUCTION}

We present in this paper the simulation structure for the MODENA project [1] and illustrates what should become an original dynamical model for the reflectivity map of the sea surface. From our point of view, MODENA challenges to find accurate models for simulating dynamically interaction between sea state, sonar, radar and ship, while keeping physical coherency during the whole observation scenario (several tens of minutes), including human participation in such a virtual reality system [3]. In such a virtual laboratory, for example, one can easily compare a SAR "Omega-K" rebuilt image resulting from a lighning by a virtual X-band radar with a picture of the same virtual scene (Fig. 1). However most of classical simulations are based on a static sea surface description [4], thus can poorly deal with dynamical properties of the interacting localised phenomena involved, nor it can deal with unpredictable behaviour of human experimenters riding a boat or a plane in the scenario. Moreover, the computation



This picture shows $4 \mathrm{~km}^{2}$ of the sea surface from a $5 \mathrm{~km}$ altitude specular point of view. For the realtime visualisation, a light spots the scene from that point of view, emissivities and diffusivities of different visualised entities (water, foam, boat) are set to constant values and an approximated geometrical optic model is computed thanks to the graphic processor unit (GPU). The sea state corresponds to the following situation : $5^{\text {th }}$ feb. 1998, 4:30 UTC, $58 \mathrm{~N}, 2 \mathrm{E}$, half an hour after the cold front of a fast low presure meteorological system. The sea state simulation involves one constant $22 \mathrm{~ms}^{-1} \mathrm{NW}$ synoptic wind, and three wave group generators: one narrow band WSW Johnswap swell $(P=16 \mathrm{~s} \pm$ $0.5 \mathrm{~s}, H_{1 / 3}=3 \mathrm{~m}$, dir $=245^{\circ} \pm 1^{\circ}$ ), and two windseas (WWNW: $P=13 \mathrm{~s} \pm 1 \mathrm{~s}, H_{1 / 3}=7 \mathrm{~m}$, dir $=280^{\circ} \pm 7^{\circ}$ and NNW: $P=8 s \pm 2 s, H_{1 / 3}=2 m$, $\operatorname{dir}=320^{\circ} \pm 10^{\circ}$ ). At this scale, only groups with wavelength greater than $50 \mathrm{~m}$ are simulated and breaking front precision is $2 m$ (smaller breaking front are not accurately simulated). Several breaking fronts (generated by groups and drawn with white cubes) exceed $100 \mathrm{~m}$ long and produce several hundreds thousands $\mathrm{m}^{3}$ of foam. In the middle of the picture, several pixels represent a $18 \mathrm{~m}$ long sailing boat.

Fig. 1. A picture of a virtual stormy sea-state by IPAS [2]

of the acoustic or electromagnetic interaction with the sea surface classically occurs on a mesh with several millions of vertex, as for example it is the case with the reflectivity map for the electromagnetic sea surface classical model; although very precise this way of computing is very slow [5]. It is thus impossible and inappropriate to compute dynamically hydrodynamic, electromagnetic and acoustic equations on an accurate grid of points or mesh, including human activity while 
the simulation occurs.

To deal with such a complexity, MODENA simulation structure aims to use an autonomy based approach: the model of each phenomenon (interaction between wave, breaking, group, ship, wind, stream, transmitter, receiver...) involved in the simulation is seen as an autonomous entity, including an autonomy of time, space and scale. In such simulation, computer activity is optimised at places where and when interaction occurs within revelant scale, according to choosen models. To be able to autonomize models for such "in virtuo" experiments, follows a methodology named enactic [6]. Enactic results from more than ten years of modeling complex systems in our multidisciplinary research center ${ }^{1}$. It has been successfully applied in biology [7]. Probably, the model used for sea state will be inspired from the IPAS model which is based on autonomized models for gravity wave group, breaking, wind, stream and depth interactions [2], because oceanography community is curious about it [8] and experiments have prouved that it can statistically be as pertinent as other more classical models [9]. Capilarity and short gravity wave, small scale wind stress, micro-breaking, electromagnetic, acoustic and hydrodynamic models will be added. Only the electromagnetic added models are briefly detailed in this paper. This modeling approach facilitates interaction between research teams involved in the MODENA project, as the whole simulation results from the set of autonomized models developed by each team in parallel.

The next section (section II) presents the methodology principles for creating models and a general overview for the resulting simulation structure. Then section III shows how enactic could lead to an original dynamical model for the reflectivity map of the sea surface. We emphase in the conclusion the risky aspect of this bet for modeling: lots of work has to be done for intricating every scale involved in remote sensing of the dynamic sea surface.

\section{FROM OBJECTS TO PHENOMENA}

We are interested, here, in defining pragmatically a constructive methodology for modeling complex systems, by using, instead of experiments, participative simulations of these models in a virtual reality system. A complex system is an open system composed of a heterogeneous set of atomic or composite entities, whose overall behavior is the result of the individual behavior of these entities and their various interactions in an active environment [10]. Because of the absence of any comprehensive behavior model for complex systems, modelisators distribute control between the components of the system and therefore autonomize the models of these components. The components could be objects (water, air, ship, radar...) or they could be interactions (hydrodynamic, aerodynamic, acoustic, electromagnetic...). Once models are created respecting the laws of the adequate fields relatively to computer abilities, virtual reality gives life to these models.

\footnotetext{
${ }^{1}$ European Center for Virtual Reality (CERV http: / / www. cerv. org) combines researchers from computer science, mathematics, physics, biology and psychology. The manifesto [3] expounds the CERV's scientific orientations: http://www.cerv.fr/en/page_pres_manifeste.php
}

The computing instrumentation of such an abstract universe, where many models interact at many scales, allows to try out the models by making them live together and with human operators, i.e. to carry out experiments in virtual reality. This in virtuo experimentation can originate a modification of the system. Man is then in enaction ${ }^{2}$ through the virtual reality system, which is created and made to evolve, through his modeling activity. Therefore, enactic [12] is a pragmatic example of methodology inspired from enaction principles giving a response to howto build such a universe of autonomized models. It emphases interaction modeling rather than object modeling.

Fistly, we introduce enactic for autonomy based modeling, then we give a rapid overview of the simulation structure for the MODENA project.

\section{A. Enactic: an autonomy based approach}

Enactic is a constructive method for studying complex systems involving multi-models and multi-scales interactions, lying on "in virtuo" simulations [12]. Enactic is based on only three assumptions and leaves a great freedom to modelisators:

1) modeling results from a human praxis (action with intention);

2) the model of a phenomenon is viewed as an autonomous entity;

3) interactions between models goes through a medium generated and made to evolve by mere models activities.

Firstly, phenomena are chosen by those who will use the virtual reality system, according to their intentions. Each modelisator will be able to try out his models interactively with those of other modelisators through the virtual reality system. Secondly, models are autonomized into autonomous entities. This assumption facilitates interaction between modelisators, as each model follows the same generic structure an thus can be developped independently. Thirdly, each phenomenon is associated to observables localised in time, space and scale. Observables are measurable properties at points of this timespace-scale by experiments. Associations of these topological points with the names of the experiments measuring properties form the experimental field for modeling the interaction medium. The experimentable properties are structures resulting from modeling action due to phenomena on their shared environment. Each model generates then dynamically the medium in function of its needs, in term of time, space and scale. Models resulting from these assumptions are rigorously formalised [13]; they are named enactic models.

Each enactic model has its own activity for acting, perceiving and reorganizing (Fig. 2). To act means for example to displace in the environment or to give localised properties. To perceive means to ask for some properties somewhere and sometimes. To reorganize means to adapt self-acting in

\footnotetext{
${ }^{2}$ Enaction: assumption according to which cognition, far from representing a given world, is the joint advent of a world and a mind starting with the history of the various actions a creature does in the world [11]. The world results from a structural coupling of the entities which create it and make it evolve, through their own activities.
} 


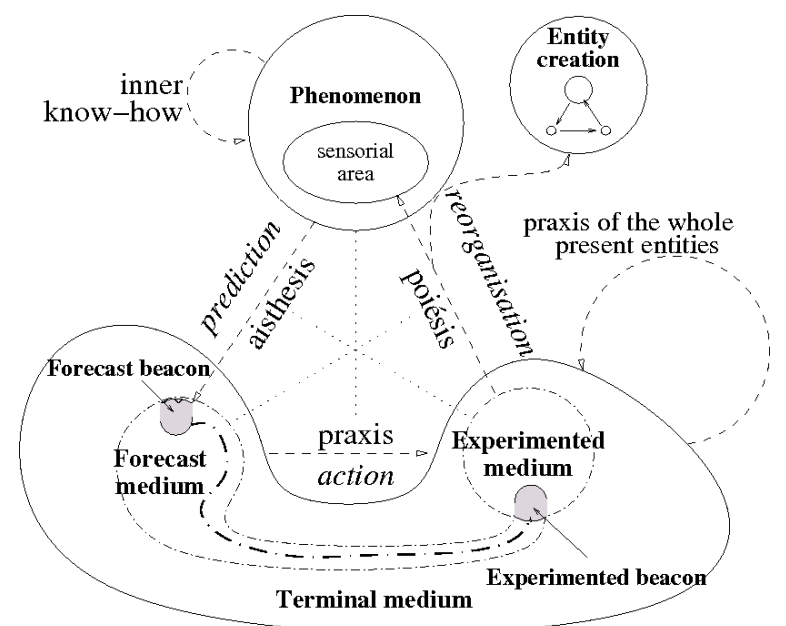

Each model for a phenomenon is characterised by a triplet (prediction, action, reorganisation) of active objects (parameters, methods and activities):

1) The prediction active object structures the interaction medium by putting beacons, localized in time, space and scale and asking for some properties, according to the perceptive needs of the phenomenon model. We call aisthesis these functions of active perception which create interaction beacons.

2) The action active object on the one hand acts at the relevant scales on the medium thus created by the whole aisthesis to give it experimented properties. We call praxis these functions making the phenomenon model perceptible by others. On the other hand, action gives autonomy to the model by executing inner know-how. 3) The reorganisation active object informs the phenomenon model about experimental results carried out in the medium generated by its aisthesis or generates another autonomized model. We call poiesis these functions for effective measurement and generation.

Fig. 2. The generic structure of enactic models

function of measured properties and also means to create new entities in the simulation for example to take care of another type of phenomenon occurring here at a given scale. Therefore, interaction medium is a dynamical structure generated and made to evolve by mere models activities.

This autonomy of time is putted alive inside the computer by chaotic asynchronous iterations (each activity sets its own time interval). Their interactions go through a medium coconstructed by mere model activity; active perceptions structure the medium, actions set its properties and reorganisations read them. This medium gives spatial and scale autonomy to entities. In such simulation, computer activity is optimised at places where and when interaction occurs. Furthermore, to add new phenomena or to modify existing models do not make previous work obsolete, as one has only to characterise interactions of new phenomena with previously defined models.

On the one hand, computer activity is naturally optimised for interaction calculus; on the other hand any conceptual interaction mismatch between one model and other ones appears very soon in the modeling construction process. Once every body agree with the virtual experimental field, we bet that interoperability is an emergent property of such a constructivistic methodology for building autonomized models.

\section{B. Simulation structure for MODENA}

The MODENA project plans to use "in virtuo" experiments for studying the remote sensing of the maritime environment. A typical scenario is during several tens of minutes and could be the following:

Some ships (including zodiacs and sailing boats) are evolving on a heterogeneous waterplane (wave groups, breakings, local winds, currents and underwater reliefs + clouds and rain), virtual and/or actual men ride them. Then a plane loading a particular radar (X-band with SAR technology, Omega-K algorithm) flies over this waterplane and modifies its trajectory according to how the "radioman" interprets radar information.

To reach that goal, the academic part of the project involves seven workpackages already oriented for interaction purposes:

1) sea-state/electromagnetic

2) object/electromagnetic

3) sea-state/object

4) radar activity/simulation resolution scale

5) antenna/plane

6) sea-state/acoustic

7) complex system simulation

The rule of the last workpackage is to intricate models proposed by previous workpackage within a complex system allowing simulations in virtual reality. So that everybody knows each others, teams have presented their models during a one day workgroup, and we met some of them a second time. We argue everybody does agree that numerical joint resolution of Navier-Stokes, Maxwell and Helmholtz equations is not an operational solution for real time simulation of the matitime environment on several square kilometers seasurface. Then, we propose to people the virtual scene by autonomised models resuming a small part of the theorical and experimental knowledge that has been acquired from hundred years of oceanography, electromagntism, naval architecture and acoustic. These specific models are not yet specified, this should be done during next year. But all of them will have to follow the formalisation for enactic models: an autonomy of time, space and scale. The choice of the appropriate scalemodel in function of computer ability is one of the major difficulty. Then, resuls from in virtuo experiments will be compared with experimental data or more classical numerical simulations.

To date, more than hundred thousand lines of code in $\mathrm{C}++$ have been written in our laboratory, making easier the implementation of enactic models in a virtual reality system thanks to the library ARéVi [14]. The ergonomics of this language has to be improved for no computer science specialists. ARéVi is a distributed virtual reality toolkit. It is both an active objectbased programming language and an execution environment. Its kernel (a group of $\mathrm{C}++$ classes) allows the creation of co-operative and distributed virtual reality applications by minimizing the programming effort. With ARéVi, a MultiAgent System (MAS) is compouned of agents (basically active objects) in an environment containing objets, eventually situated in space (2D or 3D), scale and time. ARéVi offers an 


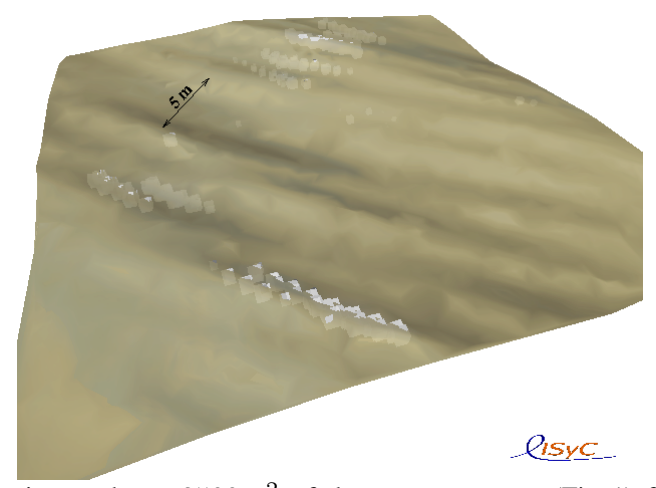

This picture shows $2500 \mathrm{~m}^{2}$ of the same sea state (Fig 1) from a $40 \mathrm{~m}$ altitude point of view. At this scale, gravity-wave groups are simulated with every wavelength greater than $50 \mathrm{~cm}$. Breaking front precision reaches $10 \mathrm{~cm}$. Small breaking fronts with a lenght lower than $5 \mathrm{~m}$ are mainly localised on crest of small waves which are themselves on the crest of bigger waves.

Fig. 3. A detailed view of the Fig. 1 sea-state

homogenous solution for interactions, implemented as method invocation or callback or message passing (point-to-point or broadcast, synchronous or asynchronous processing). ARéVi is stable and efficient. It has been used in many projects.

To start, the IPAS model for sea states could be used [2]: it respects enactic formal model and it's implemented with ARéVi. IPAS involves an oceanographical model for gravity wave groups with wavelength from several tens of centimeters to several hundreds of meters, an oceanographical models for passive and active breakings, with active fronts lenght from several centimeters to several hundreds of meters, an empirical model for wind stress over groups with dynamic Ventury effects at group scale, a descriptive model for hydrography (stream and depth fields), and a simple hydrodynamic model for ship from the zodiac to the cargo liner, including sailing boats with a decriptive model for wake and turbulence generation behind ship trajectory. Some of the whole interactions are modelled in IPAS: action towards wave groups from other groups, breakings, winds, bathymetry and currents, and action toward breakings from wave groups, winds and currents and indirectly from bathymetry through wave groups reorganisation. Interactions are computed in term of action or energy transfert, wave parameters, breaking activity, transport, refraction and creation. These enactic models assume the physical believiability of the virtual environment: action balance, wind stress, refraction and transport. Figure 1 illustrates IPAS ability to simulate a complex sea state covering $4 \mathrm{~km}^{2}$ from a point of view distant of $5 \mathrm{~km}$, while figure 3 shows the same sea state from several tens of meters and covering $2500 \mathrm{~m}^{2}$. This smaller scale simulation illustrates the ability of IPAS to describe qualitatively a laboratory observation about wind stress response to interactions between long waves and short wind-generated waterwaves [15]. Furthermore, the IPAS model has some interesting ability for generating group distribution following any desired energy spectrum [9].

We have presented the autonomy based methodology, that we plan to use for modeling remote sensing of maritime environment. Next section shows briefly how enactic could lead to an original dynamical model for the reflectivity map of the sea surface.

\section{REFLECTIVITY MAP}

To simulate dynamical aspects of microwave remote sensing for a maritime environment is a complementary approach to more conventionnal static simulations. With these last classical approaches, it takes hours and hours to simulate accurately the backscattering of the sea surface for one X-band SAR radar observation. If we want to achive dynamic simulation with human participation, you can imagine that we won't solve an Maxwell-based equation, even with an asymptotic method: we are not magicians. Nevertheless, we claim, that our method relies on the interaction between multiple simple physical models and is not only for video game or teaching purpose.

Enactic proposes to start with a review of expert knowledge about the domain of interest. Let's identify some major phenomena involved in the remote sensing of the sea surface. After that, we'll quickly expose first steps of an original model for a reflectivity map of the sea surface.

\section{A. Multiscale interactions}

To model remote sensing for a maritime environment with an aim of designing radars involves sea-states phenomena for the geometrical description of the sea surface and the related foam distribution, hydrodynamic phenomena for objects floating on the sea, electromagnetic phenomena for radar activity. What is needed for "in virtuo" microwave remote sensing experiments is a phenomenological coherence during several minutes between the sea-state, floating objects, radar virtual observations.

Sea-states mainly results from the interaction between seawaves (wind-waves, rain-ring-waves and wakes), breakings, foam, winds, currents, turbulences, shalow waters, sprays, boats, hydrocarbures, air and sea temperatures (from an oceanographic and hydrodynamic point of view). For its microwave remote sensing, one should add electromagneticwaves, active transmitors like the sun or an active antenna, receivors like an antenna, each sea-state phenomenon and may be clouds, rain drops and fog (from an electromagnetic point of view). Each phenomenon is caracterised by its time scale and its space scale:

- Time scale ranges from ten nanoseconds for the spike of an active antenna, few microseconds for boat spamming, few centiseconds for the backscatter of spikes or for boat movements, few seconds for image radar acquisition or for livespan of breacking fronts, to few minutes for long gravity wave, boat and plane trajectories; e.g: 10 magnitude orders.

- Space scale (expressed in 1D) ranges from few millimeters for small capillarity sea-waves, big drops and floating objects position, few centimeters for electromagnetic (microwave) wavelength, for large capillarity sea-waves or for micro-breakings, few meters for the 


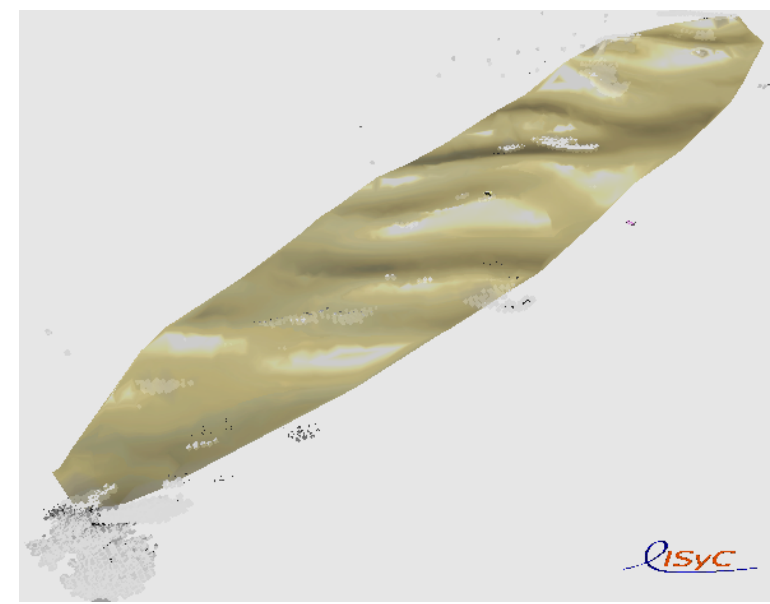

(a) Upwind lightening camera

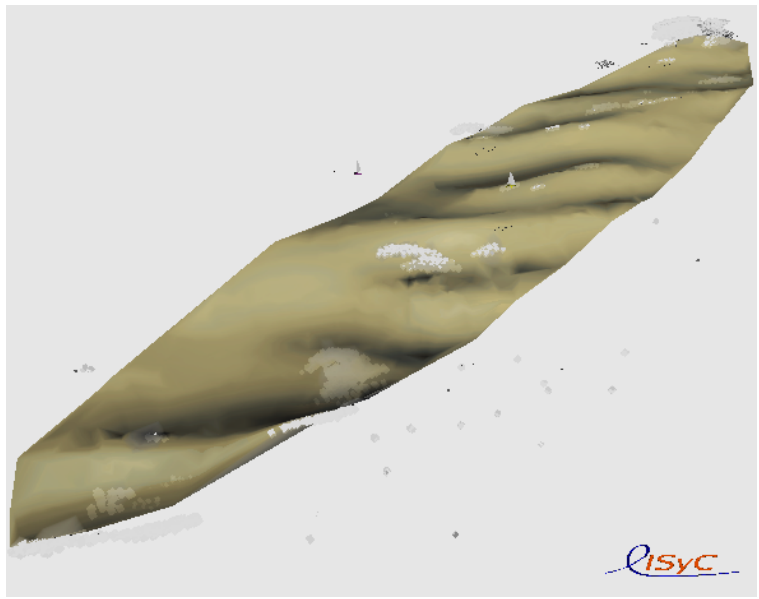

(b) Downwind lightening camera

Two cameras took one picture of the same sea-state (described in Fig. 1). The size of the enlightened sea-surface is $2 k m$ from North to South and $600 \mathrm{~m}$ from Est to West. Each camera has its own light onboard, which is ligthening only when it's taking the picture. The cameras are both with the same incidence angle, and they take the picture the one after the other. Their azimuth angles differ from $180^{\circ}$. The camera 4(a) is upwind, while the camera 4(b) is downwind. The high skewness of the two wind-seas (W-WNW and NNW) visually provides an obvious difference in the reflectivity of the sea-surface.

Fig. 4. IPAS ability for rendering the skewness of the sea slope distribution

radar resolution, for breaking, for gravity sea-waves or for floating objects length, few hectometers for longest gravity sea-waves, to few kilometers for biggest sea-wave groups, for the enlightened sea-surface side and for boat, plane and clouds trajectories; e.g: 6 magnitude orders, is 12 magnitude orders expressed in $2 \mathrm{D}$.

Besides these phenomena, theorical or empirical models have their own scales. The next example illustrates this problem for wind-sea and breaking models.

Wind-driven sea-waves are usually described by a spectrum, that gives the average height of waves mainly in function of its wavevector and wind. As the Elfouhaily directional spectrum [16] gives an accurate description for both long and short wind-driven waves, it is a good candidate for a wave generator. One should notice that, for exemple, the measurement of a sea spectrum by a bouy actually takes several hours for the convergence of the histogram, because of the spatial heterogeneity induce by the grouping mechanism. We'll need some oceanographic assumption about the distribution of groups. Moreover, a directional spectrum gives no information about the skewness of the sea slope distribution nor about sea-foam distribution, despite of their importance for scattering. To date, the sea-wave model in IPAS has some parameters for tunig the skewness in function of the wind stress over the group carrying that wave (Fig. 4), these parameters are also of major importance for the model of active breacking. A good study would be to compare IPAS abilities for generating skewness and breaking distributions according to the previous assumption about group distribution, with theorical and empirical models for the skewness of the sea slope distribution [17], for the wind stress response to interactions between long waves and short wind-generated sea-waves [15], and for active and passive sea-foam distribution [18].
As we must localise sea-state phenomena in time and space because of the needs for simulation coherence, we have to superimpose different probalistic models - mainly generators for populating environnement - with lagrangian simulations (groups, breakings, boats) in which models are autonomized because of the absence of a global model for the whole complex system. It may then be necessary to provide a backpropagation from the lagrangian simulations to the different probalistic generators. This point will be studied carefully throughout the project as it is a key for multiscale interaction.

Anyway, at each spike with a ten nanosecond duration from an active antenna, an electromagnetical simulation occurs and whatever the sea-state or floating objects models, every receivers integrates the backscattering of the environment specified by its population (the set of intanciated models in the simulation) at the time of the spike.

\section{B. Towards an enactic reflectivity map model}

The transmiter covers the sea surface by a distribution of electromagnetic beacons in function of its radiation diagram: the higher is the radiation, the closer are beacons and the thinner is their scale, a beacon is also given to each final receiver (antenna). The total number of beacons is less than 10000 per square kilometer, the average spacing between beacons is then above 10 meters; on average, each beacon deals with more than $100 \mathrm{~m}^{2}$ area. The scale $\lambda$ associated to the beacon is the rootsquare of the surface it covers. Each beacon has also a minimum of information about the transmitor : distance $d$, azimuth, incidence angle $\theta$, electromagnetic wavelength $\lambda_{e m}$, polarisation, spike description and resolution $\lambda_{\min }$.

Every entity which is in the vicinity of the beacon, and which electromagnetic action scale is greater or equal to the 
beacon scale $\lambda$ will then participate to the backscatter calculus, depending on its type (wave, foam, boat, wave-generator, micro-breaking generator...). Each of them transforms the initial spike and, thanks to asynchrone iterations, transmits it after $\delta t=d / c$ to each final receiver which cumulates every spike with a simple sum over time. One can imagine that a few entities become themselve temporary transmitters when their emissivity exceeds a given threshold.

We suppose that floating objects and largest breakings are spare enough to be instanciated, with an electromagnetic model inspired for example from [19] for ship and from [18] for large breakings with an active front longer than the beacon scale $\lambda$.

For the rough surface of sea-states (including wakes and turbulences generated by floating objects), many microwave models exists [20], [21], from the most empirical to the most computer time consuming. Composite (two scale) models for rough surface scattering are the most used [5]. They compose the specular reflexion model (sea-wavelength $\lambda_{\text {sea }}$ greater than $\left.8 \lambda_{e m}\right)$ with the Bragg resonance for $\lambda_{\text {sea }}=\lambda_{\text {em }} /\left(2 \sin \theta_{i}\right)$ at the first order, where $\theta_{i}$ is the local incidence angle due to sea-wavelength greater than $8 \lambda_{e m}$. Some assumptions on the probability of slope distribution gives average values for emissivity and simple formulas allow a fast calculus [22], but inappropriate alone with our scattered beacons for SAR resolution. We propose to use the specular model for seawavelengths $\lambda_{\text {sea }} \geq 2 \lambda$, to compute an average emissivity according to probability models related to the properties of wave-generators, cutted for $\lambda_{\text {sea }} \geq 2 \lambda$, then to modulate this average emissivity by empirical models for wave groups with $2 \lambda>\lambda_{\text {sea }}>\lambda_{\text {min }}$ acting directly as a modulation of the spike to be transmitted, with no other emissivity calculus. These small gravity wave groups would just be animated (not simulated) to reach radar resolution for Bragg resonance and small breaking, as both phenomena can be phenomenologically attached to gravity wave crests.

For the many small breakings (actif front length is between beacon scale $\lambda$ and radar resolution $\lambda_{\text {min }}$ ) and microbreakings (actif front smaller than $\lambda_{\min }$ ), we should use theorical and empirical models for active and passive sea-foam emissivity [23].

For rain perturbation on sea surface scattering [24], we suppose that an homogeneous distribution of ring-waves [25] in function of drop size [26] could be added to the Bragg resonance forgetting capillarity wave interaction in a first appriximation. Later, it may be usufull to take into account cloud and fog attenuation [27].

Thus, we have presented an outline of what could be an enactic reflectivity map model for a maritime multiscale environment. The phase computation emerges from asynchronous iterations and each entity uses a specific electromagnetic model to compute its contribution to a local emissivity. For the rough surface of sea-states a top-down mecanism onto scales allows to reach phenomenologically radar resolution while keeping coherency of wave dynamics.

\section{Conclusion}

We have presented the autonomy based methodology named enactic lying on in virtuo experiments and a general overview for the resulting simulation structure for the MODENA projet. The model of each phenomenon (wave, breaking, group, ship, wind, stream, transmitter, receiver...) involved in the simulation is seen as an autonomous entity, including an autonomy of time, space and scale. This method prefers interaction modeling than object modeling. MODENA emphases the research of models for interaction between three major groups of phenomena : sea states and electromagnetic or acoustic waves, electromagnetic or acoustic waves and objects, objects and sea states. This modeling approach facilitates interaction between research teams involved in the MODENA project, as the whole simulation results from the set of autonomized models developed by each team in parallel. The enactic models are combined and animated using multi-agents multi-scale simulation. In such simulation, computer activity is optimised at places where and when interaction occurs within revelant scale, according to choosen models.

Then, we have outlined what could be an enactic reflectivity map model for a maritime multi-scale environment. The phase computation emerges from asynchronous iterations and each entity uses a specific electromagnetic model to compute its contribution to a local emissivity. For the rough surface of seastates, a top-down mecanism onto scales allows phenomenologically to reach radar resolution while keeping the coherency of wave and foam dynamics.

Such a virtual laboratory should help for example to better distinguish the signal of breaking from the signal of small boats, in the dynamical signature of sea-states. But, lots of work has to be done for intricating every scale involved in remote sensing of the dynamic sea surface. Even if IPAS is an implemented enactic model for sea-state simulation, the electromagnetic aspects of this proposal is not implemented yet, nor the top-down mecanism onto scale; then we don't really know if it tastes as good, as it looks like. Furthermore, if that works and it should work, since we are far from the usual use of a computer for physical simulation by numerical equation solving, then we'll have to prove the physical believiability of such a simulation.

\section{ACKNOWLEDGMENT}

Thanks to Région Bretagne's and Brest Métropole Océan's financing, the MODENA project allows our laboratory (LISyC, EA 3883) to increase with one $\mathrm{PhD}$ thesis student and 6 months engineering. This would not have been possible without the partners involved in the MODENA project: ARTAL-Technologies $\odot$, BOOST-Technologies $\odot$, ENIB, ENSIETA, IETR, IFREMER $\odot$, IREENA, MAREE $\odot$, SATIMO $_{\odot}$, Telecom-Bretagne and THALES-AS $\odot$

\section{REFERENCES}

[1] R. Garello, "The MODENA project: Modeling and simulation of the maritime environment remotely sensed by radar," in Ocean'08 proceedings, Quebec City, Canada, 2008. 
[2] M. Parenthoën, T. Jourdan, and J. Tisseau, "IPAS: Interactive Phenomenological Animation of the Sea," in International Offshore and Polar Engineering Conference proceedings (ISOPE), vol. 3, Toulon, France, 2004, pp. 125-132.

[3] J. Tisseau, "Cerv's scientific manifesto," National Ingeneering School of Brest (ENIB), European Center for Virtual Reality (CERV), Brest, Tech. Rep., 2004.

[4] J. Johnson, R. Shin, J. Kong, L. Tsang, and K. Pak, "A numerical study of the composite surface model for ocean backscattering," IEEE Transactions On Geoscience And Remote Sensing, vol. 36, no. 1, pp. 72-83, 1998.

[5] D. Lemaire, P. Sobieski, C. Craeye, and A. Guissard, "Two-scale models for rough surface scattering: Comparison between the boundary perturbation method and the integral equation method," Radio Science, vol. 37, no. 1, pp. 10.1001-10.1029, 2002.

[6] M. Parenthoën and J. Tisseau, "Enactic applied to sea state simulation," in Enactive'06 proceedings, 2006.

[7] G. Desmeulles, G. Querrec, P. Redou, S. Kerdélo, L. Misery, V. Rodin, and J. Tisseau, "The virtual reality applied to biology understanding: the in virtuo experimentation," Expert Systems with Applications, vol. 30 , no. 1, pp. 82-92, 2006.

[8] M. Parenthoën, "Enactive modeling for natural phenomena - example of sea state simulations," in Physical Oceanography Dissertation Symposium Abstract (PODS III), Hawaii, 2005, pp. 18-19.

[9] C. Le Gal, M. Olagnon, M. Parenthoën, P.-A. Béal, and J. Tisseau, "Comparison of sea state statistics between a phenomenological model and field data," in Ocean'07 proceedings, 2007.

[10] E. Morin, Introduction à la pensée complexe. Paris: ESF Editeur, 1990.

[11] F. Varela, E. Thompson, and E. Rosch, The embodied mind: cognitive science and human experience. Cambridge: MIT Press, 1991.

[12] M. Parenthoën and J. Tisseau, Tutorial book of virtual concept. Biarritz, France: ENSIAME-LAMIH/LIPSI-ESTIA, 2005, ch. Enactive modeling.

[13] M. Parenthoën, "Animation phénoménologique de la mer - une approche énactive —," Ph.D. dissertation, Université de Brest (UBO), Lab. d'Informatique des Systèmes Complexes (LISyC - EA 3883), Centre Européen de Réalité Virtuelle (CERV), Brest, France, 2004.

[14] F. Harrouet, E. Cazeaux, and T. Jourdan, Le traité de la réalité virtuelle 3rd ed. Les Presses de l'Ecole des Mines de Paris, 2006, vol. 3: Outils et modèles informatiques des environnements virtuels, ch. ARéVi, pp. 369-392.

[15] V. Makin, H. Branger, W. Peirson, and G. J-P., "Modelling of laboratory measurements of stress in the air flow over wind-generated and paddle waves," Journal of Physical Oceanography, vol. 37, no. 12, pp. 2824 2837, 2007.
[16] T. Elfouhaily, B. Chapron, K. Katsaros, and D. Vandemark, "A unified directional spectrum for long and short wind-driven waves," Journal of Geophysical Research, vol. 102, no. C7, pp. 15.781-15.796, 1997.

[17] B. Chapron, D. Vandemark, and T. Elfouhaily, "On the skewness of the sea slope probability distribution," Geophysical monograph, vol. 127, pp. 59-63, 2002.

[18] N. Reul and B. Chapron, "A model of sea-foam thickness distribution for passive microwave remote sensing applications," Journal of Geophysical Research, vol. 108, no. C10, pp. 19.1-19.14, 2003.

[19] E. Shearman, "A review of propagation and scattering factors in remotesensing and ship-tracking by HF (High Frequency) radar," in $A G A R D$ Propagation Factors Affecting Remote Sensing by Radio Waves (SEE N84-15646 06-43), G. Millman, Ed., 1983, p. 13.

[20] A. Fung, Microwave Scattering and Emission Models and their Application. Norwood, Mass.: Artech Hous, 1994.

[21] A. Voronovitch, Wave Scattering From Rough Surface. New York: Springer-Verlag, 1994.

[22] Y. Liu, M.-Y. Su, X.-H. Yan, and W.-T. Liu, "The mean-square slope of ocean surface waves and its effects on radar backscatter," Journal of Atmospheric and Oceanic Technology, vol. 17, pp. 1092-1105, 2000.

[23] A. Camps, M. Vall-llossera, R. Villarino, N. Reul, B. Chapron, I. Cordella, N. Duffo, F. Torres, J. Miranda, R. Sabia, A. Monerris, and R. Rodríguez, "The emissivity of foam-covered water surface at L-band: theoretical modeling and experimental results from the Frog 2003 field experiment," IEEE Transaction On Geoscience And Remote Sensing, vol. 43, no. 5, pp. 925-937, 2005.

[24] M. Bulatov, G. Bolotnikova, V. Etkin, and E. Skvortsov, "Investigation of microwave scattering and emission of sea surface perturbation by rain conducted with space "ALMAZ-1" SAR and shipborne radiometers," in International Geoscience and Remote Sensing Symposium (IGARSS'95). 'Quantitative Remote Sensing for Science and Applications'. IEEE CNF, 1995, pp. 1078-1080.

[25] D. Lemaire, L. Bliven, C. Craeye, and P. Sobieski, "Drop size effects on rain generated ring-waves with a view to remote sensing applications," International Journal of Remote Sensing, vol. 23, no. 12, pp. 2345-2357, 2002.

[26] Y. Furuhama and T. Ihara, "Remote sensing of path-averaged raindrop size distributions from microwave scattering measurements," IEEE Transactions on Antennas and Propagation, vol. AP-29, pp. 275-281, 1981.

[27] A. Berne and R. Uijlenhoet, "Quantitative analysis of X-band weather radar attenuation correction accuracy," Natural Hazards and Earth System Sciences, vol. 6, pp. 419-425, 2006. 